\title{
Enhanced Ice Nucleation and Growth by Porous Composite of rGO and Hydrophilic Silica Nanoparticles
}

Haoran Liang ${ }^{1}$, Ottmar Möhler ${ }^{2}$, Steven Griffiths ${ }^{1}$ and Linda Zou ${ }^{1 *}$

${ }^{1}$ Department of Civil Infrastructure and Environment Engineering, Masdar Institute, Khalifa University of Science and Technology, Abu Dhabi, United Arab Emirates

${ }^{2}$ Institute of Meteorology and Climate Research, Karlsruhe Institute of Technology, Eggenstein Leopoldshafen, Germany

*Correspondence author: linda.zou@ku.ac.ae (Prof. Linda Zou)

This PDF file includes:

Video S1. Real-time ice nucleation process of the PrGO-SN composite (rough regions).

Video S2. Real-time ice nucleation process of the PrGO-SN composite (smooth regions).

Note that these videos were corresponding to Figure $5 \mathrm{c}$ and $5 \mathrm{~d}$ in the main manuscript and were played at a $x 4$ rate.

S-I. Experiment and characterization information.

Figure S1. Comparison of water vapor adsorption capacity of different PrGO-SN composites. Figure S2. STA analysis of the relative mass ratio of the PrGO-SN composite.

Figure S3. Additional E-SEM images of ice nucleation activities of the PrGO-SN composite. 


\section{S-I Experiment and characterization information}

Materials. Tetraethyl orthosilicate ( $\geq 99.0 \%$, TEOS) and ammonium hydroxide solution (30 \% $\left.33 \%, \mathrm{NH}_{3} \cdot \mathrm{H}_{2} \mathrm{O}\right)$ were purchased from Sigma Aldrich. Graphene oxide $(2 \mathrm{mg} / \mathrm{ml})$ were synthesized in the lab according to the modified Hummer method as reported. Deionized (DI) water was used in all experimental processes.

Synthesis of $\operatorname{PrGO}-\mathrm{SN}$ composite. A single-step hydrothermal synthesis was carried out with following details: $15 \mathrm{ml} \mathrm{GO}$ solution ( $2 \mathrm{mg} / \mathrm{ml}$ concentration) prepared above was mixed with 20 $\mathrm{ml}$ ethanol, then $0.7 \mathrm{ml} \mathrm{NH} 3 \cdot \mathrm{H}_{2} \mathrm{O}$ and $0.7 \mathrm{ml}$ TEOS were added into the mixture. The mixture was sonicated for $30 \mathrm{~min}$, then transferred into a sealed Teflon-lined autoclave and heated hydrothermally at $180{ }^{\circ} \mathrm{C}$ for $12 \mathrm{~h}$. After hydrothermal process, formed PrGO-SN composite was washed with DI water three times, then put into a freezer at $-20{ }^{\circ} \mathrm{C}$ overnight. Subsequently, the composite was transferred into freeze dryer at $-100^{\circ} \mathrm{C}$ for $24 \mathrm{~h}$.

Characterization and measurements. The synthesized PrGO-SN composite were characterized through Scanning Electron Microscopy (SEM, Quanta 250, FEI Company); Transmission Electron Microscopy (TEM, Tecnai from FEITM Company operating at $200 \mathrm{KV}$ ); A Fourier transform infrared Nicolet iS10 spectrometer (FTIR, Thermo Fisher Scientific, Inc.). Water static-contactangle Measurements (Kyowa DM-701) elaborated with an interface Measurement \& Analyses System and the droplets of $0.8 \mu \mathrm{l}$. The water vapor adsorption performance of the PrGO-SN composite was determined quantitatively via a water-vapor adsorption isotherm test (BrunauerEmmett-Teller, Belsorb Max, Japan) in water-vapor mode. Note that the samples were pretreated at $100{ }^{\circ} \mathrm{C}$ for 3 hours under a pressure $<10^{-4} \mathrm{~Pa}$ before commencing the analysis and the whole test required 24 hours. Environmental scanning electron microscopy (E-SEM) was used for in-situ observation of the ice nucleation activities of the PrGO-SN composite. 


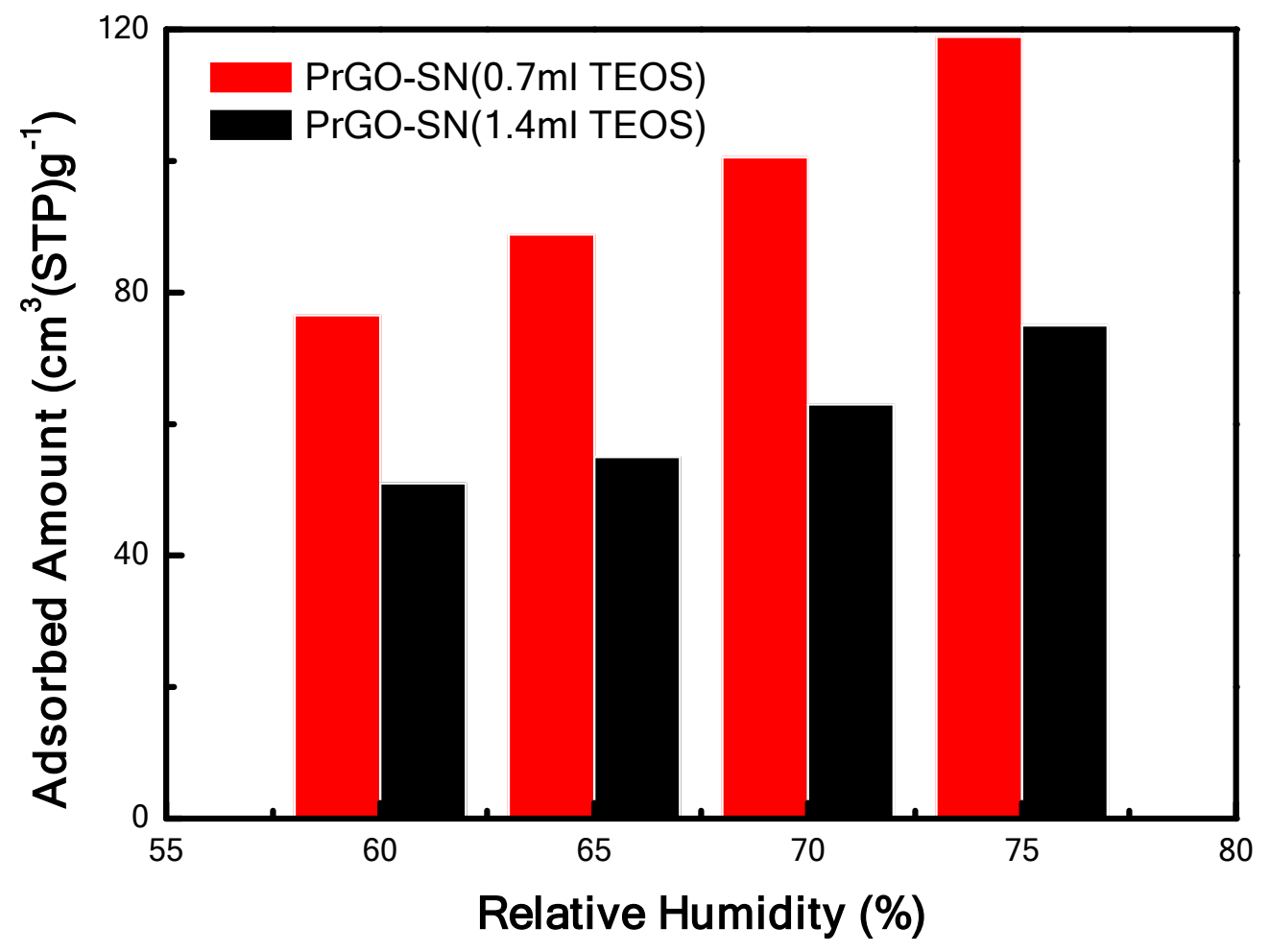

Figure S1. Comparison of water vapor adsorption capacity of different PrGO-SN composites. At low relative humidity conditions ranging from $60 \%$ to $75 \%$, the adsorbed water-vapor volume of the PrGO-SN(0.7ml TEOS) sample was higher than that of the PrGO-SN(1.4ml TEOS) sample; as the relative humidity increases, the difference in water vapor adsorption capacity was even larger. 


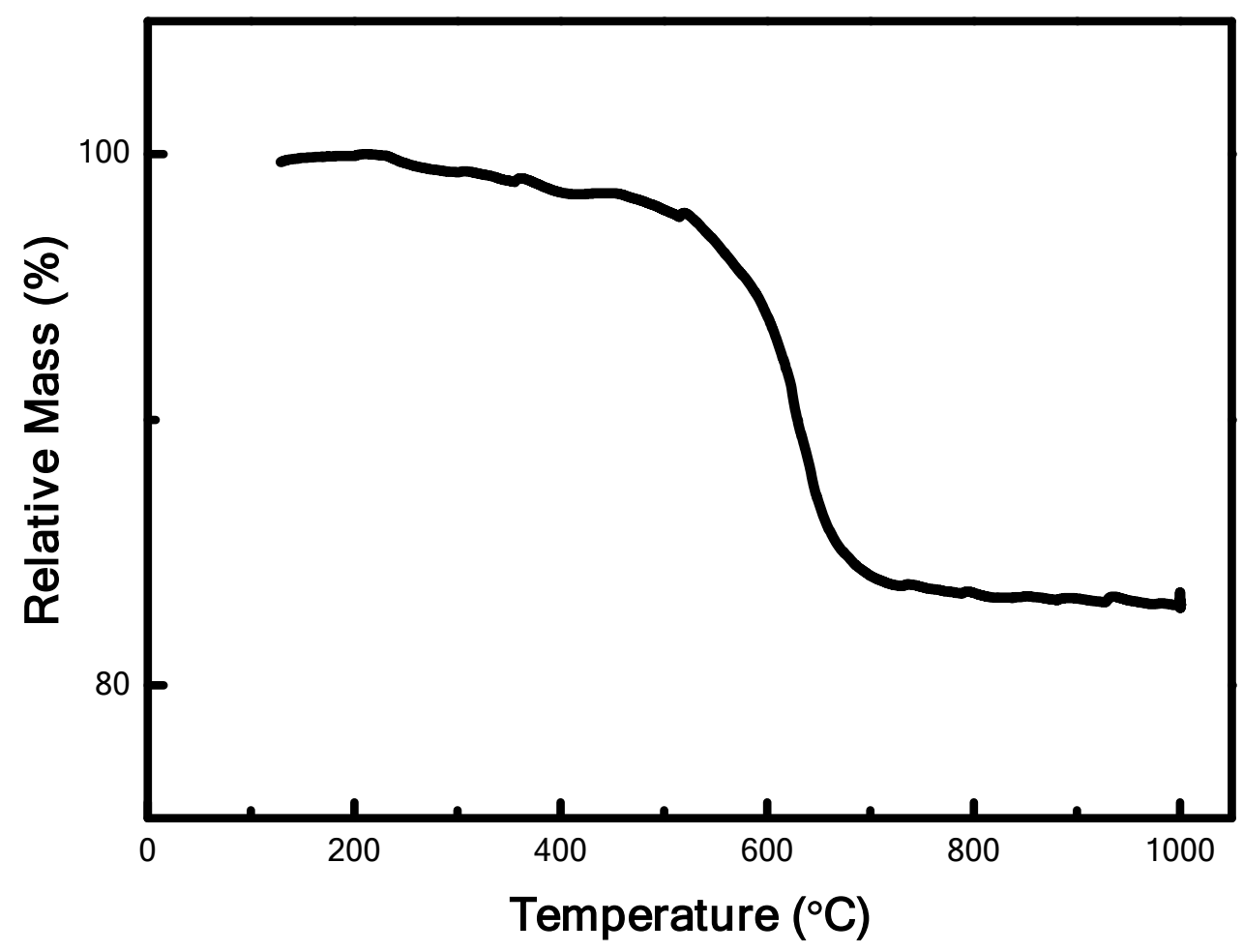

Figure S2. Simultaneous thermal analysis (STA) of the relative mass ratio of the PrGO-SN composite. Results indicated that $\mathrm{rGO}$ accounted for $18 \%$ of the total composite mass. Typically, the mass of composite did not change much until $500{ }^{\circ} \mathrm{C}$. Before $500{ }^{\circ} \mathrm{C}$, most of the mass loss was due to the evaporation of water molecules inside the composite; after $500{ }^{\circ} \mathrm{C}$, carbonaceous materials started to burn, resulting in significant mass loss. 

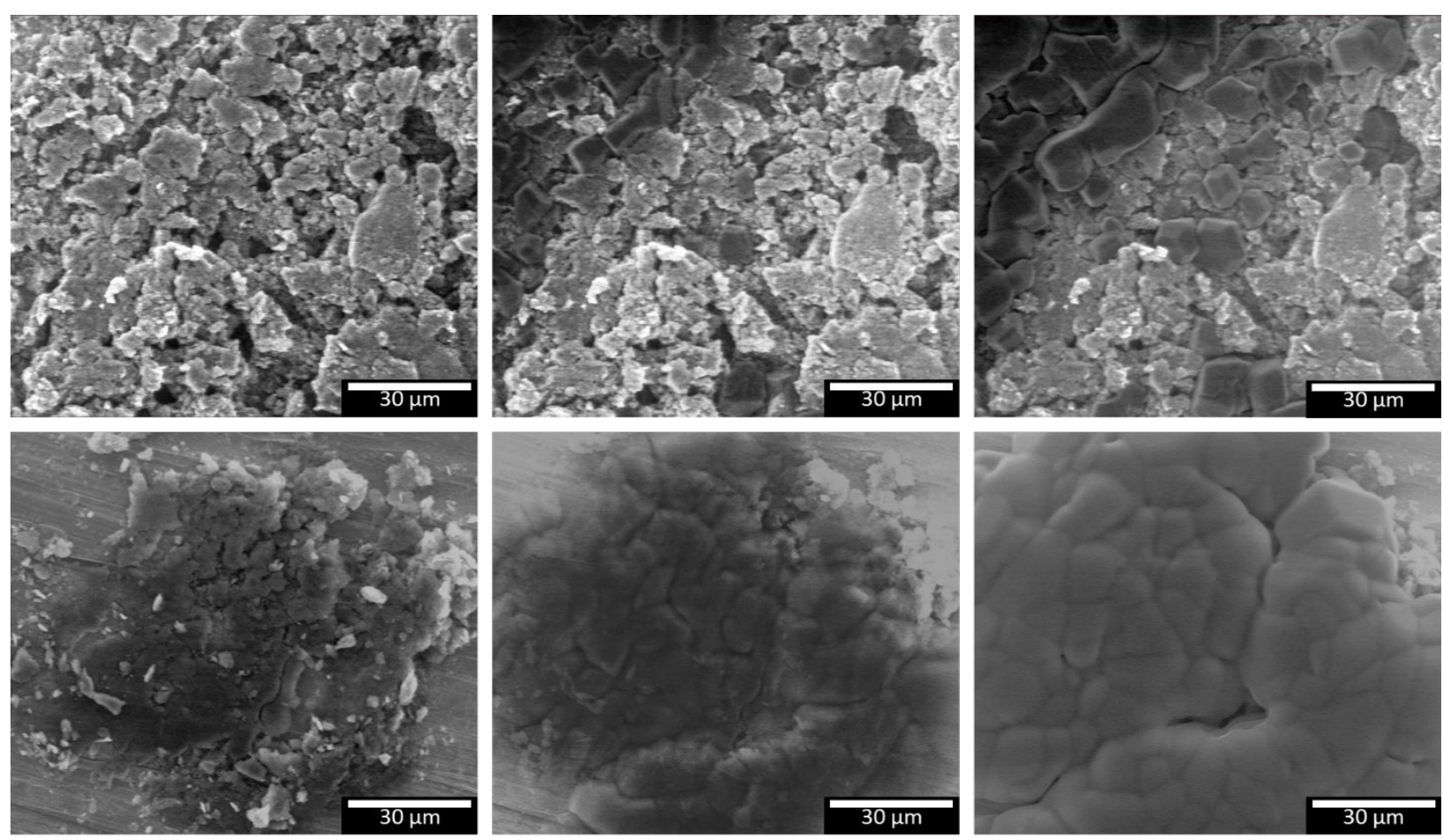

Figure S3. Additional E-SEM images of ice nucleation activities of the PrGO-SN composite. E-SEM experiments showed different ice formation patterns in rough region with cavities (top) and relatively smooth region (bottom) in form of hexagonal ice crystals and bulk ice, respectively; the scale bars are $30 \mu \mathrm{m}$ for all images. 